\title{
Criminologie
}

\section{Introduction : la trame des parcours délinquants}

\section{Pierre Tremblay et Carlo Morselli}

Volume 37, numéro 2, automne 2004

Délinquance et réussite

URI : https://id.erudit.org/iderudit/010702ar

DOI : https://doi.org/10.7202/010702ar

Aller au sommaire du numéro

Éditeur(s)

Les Presses de l'Université de Montréal

ISSN

0316-0041 (imprimé)

1492-1367 (numérique)

Découvrir la revue

Citer ce document

Tremblay, P. \& Morselli, C. (2004). Introduction : la trame des parcours délinquants. Criminologie, 37(2), 3-8. https://doi.org/10.7202/010702ar d'utilisation que vous pouvez consulter en ligne.

https://apropos.erudit.org/fr/usagers/politique-dutilisation/ 


\title{
Introduction: la trame des parcours délinquants
}

\author{
Pierre Tremblay \\ École de criminologie \\ Université de Montréal \\ pierre.tremblay@umontreal.ca \\ Carlo Morselli \\ École de criminologie \\ Université de Montréal \\ carlo.morselli@umontreal.ca
}

Certaines trajectoires délinquantes sont brèves et se résument parfois à un seul passage à l'acte implosif. D'autres s'étalent sur une longue période et sont ponctuées de succès plus ou moins temporaires et de revers plus ou moins décisifs. Certains délinquants, qu'ils soient juvéniles ou adultes, «réussissent» mieux ce parcours que d'autres. Ces réussites délinquantes ${ }^{1}$ constituent le thème de cette livraison.

Il nous a semblé utile d'ouvrir ce numéro en présentant l'article que Michel Kokoreff consacre aux filières d'importation de cannabis depuis le Maroc via l'Espagne jusqu'en France, durant les années 1990, et de le clore avec l'étude que Julie Paquin consacre au marché des factures de complaisance dans l'industrie du vêtement à Montréal durant les années 1970.

1. Par réussite délinquante, on entend toute séquence de délits au terme de laquelle les délinquants qui y participent peuvent légitimement se persuader (et persuader d'autres délinquants) qu'ils ont utilisé de manière efficace les moyens ou les ressources qui étaient à leur disposition pour parvenir à leurs fins. Dans ce numéro, les réussites des délinquants sont évaluées au regard de leur aptitude à gagner de l'argent et les séquences délictuelles sont étalées sur 36 mois et plus. 
Les deux articles prennent la forme d'études de cas qui exploitent la richesse des dossiers d'enquête et des archives judiciaires pour formuler des hypothèses originales: celle de la reconversion du grand banditisme dans les trafics de stupéfiants depuis une vingtaine d'années (Kokoreff) et celle de la diffusion concomitante des pratiques de délinquance d'affaires dans les trafics de véhicules volés (Paquin). De telles études de cas sont précieuses parce qu'elles permettent de formuler des hypothèses précises sur les changements qualitatifs de la criminalité et les innovations délinquantes (ou les réussites collectives) qui en sont à l'origine.

On notera également que ces deux études de cas s'attardent tout particulièrement aux problèmes spécifiques que les réussites délinquantes posent aux tribunaux criminels. Kokoreff se penche sur la rareté des procédures pénales qui visent à indexer l'échelle des peines en fonction «non plus des risques que le trafic fait courir aux victimes potentielles mais de son degré d'organisation», c'est-à-dire son degré de réussite. À l'inverse, Paquin se penche sur «l'anomalie apparente de l'avalanche des poursuites pénales et civiles » déclenchées qui s'est produite dans une cause de fraude, dont l'on dispose habituellement de manière beaucoup plus discrète et inoffensive. C'est en analysant ces exceptions à la règle (les «faux positifs») que les études de cas donnent le meilleur d'ellesmêmes.

Le thème de ce numéro recoupe celui que la revue Criminologie avait consacré, en 1989, au «milieu criminel». Mais on appréciera le chemin parcouru en remarquant que quatre des articles thématiques procèdent par voie de sondage de délinquance révélée et s'attardent moins aux succès de certaines "pratiques délinquantes» qu'aux trajectoires individuelles de ceux qui y participent.

Clément Robitaille réanalyse l'enquête que la Rand Corporation avait réalisée en 1978 auprès d'un échantillon important de détenus américains. On y trouve en effet des questions précises (mais jamais analysées) sur leurs revenus illicites durant les trois années qui avaient précédé leur incarcération. Son mérite est d'apporter plusieurs éléments de réponse, parfois surprenants, à la question : Pourquoi certains délinquants sontils plus efficaces que d'autres?

Il établit tout d'abord que les délinquants qui s'abstiennent de violence retirent de leurs délits des revenus moins élevés que ceux qui en font un usage stratégique. Nonobstant le bonnet de «rationalité limitée» dont les criminologues se plaisent à coiffer les délinquants, Robitaille montre aussi que les réussites délinquantes sont généralement «méri- 
tées». Elles ne sont le fruit ni du hasard ni de la chance. Plus les délinquants «travaillent» leurs délits et en soignent l'exécution, plus ils en tirent des bénéfices tangibles. Il montre enfin que les délinquants qui sont les plus enclins à la récidive ne sont pas nécessairement les moins doués d'entre eux mais tout aussi bien ceux qui connaissent un succès enviable dans leurs activités illicites. Une nouvelle condamnation représente sans doute un revers, mais elle peut être aussi un indice présomptif de réussite (et de persévérance). Cela explique, entre autres choses, qu'il n'est pas si «difficile» de trouver en prison un nombre appréciable de délinquants performants.

Mathieu Charest fait état des inégalités de performance (et de revenus) qui se dégagent d'un sondage auprès d'un échantillon de détenus fédéraux canadiens réalisé en 2000-2001. Des 262 sujets qui composent cet échantillon, un premier tiers se disqualifie d'emblée, en ce sens que les délits n'avaient pas pour finalité de procurer un avantage économique; le deuxième tiers regroupe un ensemble de délinquants qui en moyenne retirent bon an mal an 25000 \$ de leurs délits, alors que le dernier tiers se subdivise en deux classes d'importance équivalente: un sixième des détenus atteignent en moyenne la barre des 90000 \$ par année et le dernier sixième, le cap des $350000 \$$. On est assez loin de Jean Valjean et des Misérables. Il est difficile de penser que ces réussites délinquantes ne constituent pas en soi un puissant facteur de «motivation» ou d'émulation pour bon nombre de délinquants potentiels.

Mais peut-on se fier aux délinquants pour nous renseigner sur les bénéfices de leurs délits ou les résultats de leur performance? Charest propose un inventaire des stratégies que les chercheurs utilisent pour statuer sur la validité des revenus que les délinquants déclarent retirer de leurs activités illicites. La stratégie de base qui donne lieu à de nombreuses variantes est de poser aux sujets deux questions ou deux séries de questions qui ont la même fonction, mais qui different sous quelque rapport, et de trianguler par la suite les deux mesures de revenus. Les résultats montrent que les discordances observées sont d'ordre cognitif et attribuables à la complexité intrinsèque des opérations de calcul mental qu'elles exigent des sujets. Cela amène Charest à trouver une solution efficace pour corriger l'imprécision des mesures et à faire augmenter le coefficient de concordance des deux mesures de revenus de 0,45 à 0,92 .

Dans leur article, Morselli et Tremblay développent la thèse selon laquelle le capital social des délinquants leur permet d'augmenter de 
manière significative la qualité de leur performance. Pour mesurer ce capital social, il faut d'abord reconstituer dans le détail le réseau des relations des délinquants (leur réseau «égocentrique» de co-délinquance) et puis l'analyser pour en déterminer l'efficacité potentielle. L'approche s'inspire des travaux en sociologie du travail qui ont su démontrer que les chances de promotion des employés des grandes entreprises étaient inversement proportionnelles au degré de redondance de leur réseau de relations. Les individus les moins performants auraient un réseau de relations «tricoté serré» (ou redondant) et les plus performants, un réseau «riche en trous structuraux». Ce qui vaut pour les jeunes adultes à la recherche d'un emploi ou pour les cadres des compagnies devrait également valoir pour les délinquants. En montrant que c'est bien le cas, cette étude détruit en même temps le mythe selon lequel les délinquants seraient par définition des «ratés», en raison de leur présentisme ou de la labilité ${ }^{2}$ de leur «caractère».

Plus les délinquants sont performants, moins ils s'investissent dans le marché du travail (Morselli et Tremblay). Cette relation peut se décliner inversement. Ceux qui ont peu d'espoir de trouver leur compte dans les emplois conventionnels à leur portée devraient être davantage motivés à investir leurs énergies dans la délinquance. Bon nombre de criminologues estiment qu'il n'y a pas de relation causale entre le besoin d'argent et le penchant à la délinquance. Mais cette question a le plus souvent été traitée de manière empiriquement désinvolte.

Ce n'est pas le cas de l'article de Bill McCarthy et John Hagan. Les données proviennent d'une enquête de terrain auprès d'un échantillon de «jeunes de la rue», réalisée en 1987 à Toronto. Le mérite de cette étude est de chiffrer minutieusement le montant des allocations familiales et revenus d'emploi des sujets, alors qu'ils n'avaient pas encore déserté leur famille et d'analyser ensuite l'impact du besoin d'argent sur la fréquence de leurs délits. L'effet attendu est bel et bien observé : plus leurs revenus personnels sont faibles, plus les sujets sont actifs dans la délinquance. Mais cet effet est opérant seulement pour les plus démunis d'entre eux (l'utilité marginale d'un supplément de revenu étant

2. Maurice Cusson (1998: 86) souligne fort à propos que la notion de labilité introduite par Jean Pinatel, en 1963, a été «réinventée» par Gottfredson et Hirschi (1990) qui l'ont baptisée low self-control (faiblesse de caractère). Par labilité, Pinatel entendait une «combinaison d'imprévoyance, d'inorganisation dans la durée et d'instabilité du caractère». L'enthousiasme avec lequel la communauté criminologique anglo-saxonne a reçu cette théorie en dit long, malheureusement, sur le déclin de la sociologie criminelle. 
marginalement décroissante), seulement chez les garçons, et seulement pour les formes congruentes de délinquance (les délits contre les biens et les petits trafics, mais pas les délits de violence ou la toxicomanie). Une relation causale n'a pas à être rejetée sous prétexte que sa véracité est «conditionnelle» à des effets de seuil ou de pertinence. Un des mérites de cette analyse est d'avoir montré que des résultats apparemment contradictoires peuvent être concrètement résolus en opérationnalisant de manière explicite la notion d'utilité marginale (chère aux économistes) dans les analyses des choix délinquants.

Une dernière remarque, en guise de conclusion. En renonçant à analyser la performance des délinquants persistants, la sociologie criminelle s'est marginalisée. Elle gagnerait, nous semble-t-il, à puiser au contraire ses données chez les délinquants qui se sont effectivement confrontés aux pouvoirs publics.

Les articles hors thème dans ce numéro apportent, de leur côté, une réflexion sur deux questions de fond en criminologie.

D'une part, Denis Lafortune et Richard Lusignan, dans une situation où il est question de créer au Québec un ordre professionnel des criminologues, mettent en perspective le contexte de travail de ces derniers. En effet, les pratiques dans le domaine de la criminologie surviennent dans un contexte d'autorité, telles l'administration de la justice pénale ou l'intervention clinique. Ils soulignent alors que, compte tenu de ce cadre de travail, des enjeux liés à la confidentialité de leurs interventions ou encore au pouvoir important accordé à l'intervenant font que la réflexion non seulement sur la formation universitaire mais aussi sur le contrôle éthique/déontologique que cette profession nécessite est essentielle.

D'autre part, Gilles Chantraine apporte un certain regard sur le monde carcéral à travers une analyse des relations de pouvoir entre surveillants et détenus d'une prison française. Son point de départ est la construction d'une typologie selon la place des détenus dans le cadre de leur détention, stratège, tacticien, soumis. L'auteur montre alors que, entre privilèges, négociation et obéissance, des rapports de contre-pouvoir s'instaurent. Ceux-ci amènent un ordre négocié marqué par une hétérogénéité des relations au sein desquelles les capacités de résistance des personnes incarcérées varient largement. 


\section{Références}

Cusson, M. (1998). Criminologie actuelle. Paris: Presses Universitaires de France.

Gottfredson, M., \& Hirschi, T. (1990). A general theory of crime. Stanford: Stanford University Press. 